\title{
Hyperpolarization-activated cation currents in medium-size dorsal root ganglion cells are involved in overactive bladder syndrome in rats
}

Chao Tan ${ }^{1 \dagger}$, Fei Yan ${ }^{1 \dagger}$, Li-Ping Yao ${ }^{2 \dagger}$, Jun-Ling Xing ${ }^{3 \dagger}$, Wei-Jun Qin ${ }^{1}$, Kun Zhang ${ }^{1}$, Guo-Jun Wu ${ }^{1}$, Jian-Lin Yuan ${ }^{1 *}$ and Fei Liu ${ }^{1 *}$ (D)

\begin{abstract}
Background: To investigate the functions of the hyperpolarization-activated cation currents in medium-size dorsal root ganglion cells in a rat model of overactive bladder syndrome.

Methods: Rats with OAB were screened using a urodynamic testing device. The whole-cell patch clamp technique was used to investigate changes in excitability and hyperpolarization-activated cation current $\left(l_{h}\right)$ of medium-size cells in the L6 dorsal root ganglia (DRG) of the OAB rats. Intrathecal injection of the specific $/ \mathrm{h}$ inhibitor ZD7288 was used to investigate changes of voiding function and $I_{h}$ of medium-size cells in the L6 DRG.

Results: The urinary bladder weight of the $O A B$ rats was significantly increased $(p<0.01)$; However, 7 days after intrathecally administration of ZD7288 $(2 \mu \mathrm{M})$, the weight of rat bladder was significantly reduced $(p<0.01)$. The excitability of the medium-size cells in the L6 DRG of the OAB rats was significantly increased, and the number of action potentials elicited by a 500 pA stimulus was also markedly increased. Furthermore, ZD7288 significantly reduced the excitability of the medium-size DRG cells. The medium-size cells in the DRG of the OAB rats had a significantly increased $I_{h}$ current density, which was blocked by ZD7288.
\end{abstract}

Conclusions: The $I_{h}$ current density significantly increased in medium-size cells of the L6 DRG in the OAB model. A decrease of the $I_{h}$ current was able to significantly improve the voiding function of the OAB rats, in addition to lowering their urinary bladder weight. Our finding suggested that the observed increase of $I_{\mathrm{h}}$ current in the medium-size DRG neurons might play an important role in the pathological processes of OAB.

Keywords: Overactive bladder, $I_{h}$, Dorsal root ganglion, Hyperpolarization, ZD7288

\footnotetext{
* Correspondence: jianliny@fmmu.edu.cn; liufeiyh@163.com

${ }^{\dagger}$ Chao Tan, Fei Yan, Li-Ping Yao and Jun-Ling Xing contributed equally to

this work.

'Department of Urology, Xijing Hospital, Air Force Medical University, 15

Changle West Road, Xi'an 710032, Shaanxi, China

Full list of author information is available at the end of the article
}

C C The Author(s). 2020 Open Access This article is licensed under a Creative Commons Attribution 4.0 International License, which permits use, sharing, adaptation, distribution and reproduction in any medium or format, as long as you give appropriate credit to the original author(s) and the source, provide a link to the Creative Commons licence, and indicate if changes were made. The images or other third party material in this article are included in the article's Creative Commons licence, unless indicated otherwise in a credit line to the material. If material is not included in the article's Creative Commons licence and your intended use is not permitted by statutory regulation or exceeds the permitted use, you will need to obtain permission directly from the copyright holder. To view a copy of this licence, visit http://creativecommons.org/licenses/by/4.0/ The Creative Commons Public Domain Dedication waiver (http://creativecommons.org/publicdomain/zero/1.0/) applies to the data made available in this article, unless otherwise stated in a credit line to the data. 


\section{Background}

Overactive bladder syndrome $(\mathrm{OAB})$ is characterized by urinary urgency accompanied by frequent urination and nocturia, with or without urinary urge incontinence [1]. $O A B$ severely affects the patients' quality of life, since frequent urination can cause severe inconvenience, whereas long-term incontinence can lead to urinary tract infections, possibly leading to severe clinical complications affecting the patient's physical and mental wellbeing [2]. Therefore, the micturition reflex which is subject to complex control from both the central and the peripheral nervous system, is attracting more attention. Anatomic studies have uncovered that the relevant afferent nerves mainly pass through the pelvic posterior, mainly from the L6 and S1 dorsal root ganglia (DRG) to the spinal cord, to trigger the micturition reflex [3]. According to the diameter of the neurons, the afferent DRG nerve fibers are classified as large, medium and small, which are further categorized into $A_{\alpha / \beta}, A_{\delta}$ and Ctype fibers $[4,5]$. The afferent nerves controlling of the urinary bladder include the myelinated $\mathrm{A}_{\delta}$ and the unmyelinated C-type fibers [6]. The hyperpolarizationactivated cyclic nucleotide-gated cation ( $\mathrm{HCN}$ ) currents $\left(I_{\mathrm{h}}\right)$ have been reported to be expressed by many cell types, such as cardiac pacemaker cells and retinal photoreceptor cells, as well as central and peripheral nerve cells, including DRG cells.

It has been discovered that small- and medium-size nerve cells of the DRG which can control urinary bladder all possess $I_{\mathrm{h}}$ currents, and the $I_{\mathrm{h}}$ current of the medium-sized neurons is significantly higher than that of the small neurons [7]. This indicates that the $I_{\mathrm{h}}$ current is predominantly present in the medium-size DRG neurons. Even though the afferent neurons that control the bladder include $\mathrm{C}$ and $\mathrm{A}_{\delta}$ type fibers, in normal physiological conditions, the $\mathrm{A}_{\delta}$ fibers are solely responsible for the mechanical nociceptive reflex when the bladder expands to threshold pressure. This indicates that the medium-size neurons of the DRG play an important role in the micturition reflex. The $I_{\mathrm{h}}$ current activated by hyperpolarization of the $\mathrm{HCN}$-gated channels has been shown to be involved in many important physiological functions, including the pacemaker function of the heart and brain as well as maintaining the resting membrane potential and cell excitability $[8,9]$.

Masuda and coworkers have discovered that ZD7288, a specific inhibitor of $\mathrm{HCN}$-gated channels, can inhibit the $I_{\mathrm{h}}$ current in the medium-size cells that control the bladder, markedly increasing the duration of the afterhyperpolarization potential of an action potential [7]. This in turn implies that the $I_{\mathrm{h}}$ current influences the excitability of the medium-size DRG neurons that innervate the bladder. In order to find out the relationship between $\mathrm{OAB}$ and the $I_{\mathrm{h}}$ current in medium-size DRG neurons, this study was performed to characterize the electrophysiological properties of the $I_{\mathrm{h}}$ current in the medium-size cells of the L6 dorsal root ganglion in a rat model of overactive bladder, and to investigate its influence on the model animals' urodynamics.

\section{Methods \\ Experimental animals}

Experiments were performed using adult female SD rats, aged $7-8$ weeks, weighing $200-220$ g, provided by the Air Force Medical University animal facility. Under standard experimental conditions, the animals were kept on a $12 \mathrm{~h}$ day and night cycle, at $25^{\circ} \mathrm{C}$, and were provided with rat chow (Allentown, USA) and water ad libitum. The rats were allowed to acclimatize to the laboratory environment for 1 week prior to starting the experiments. The experiments were approved by the Air Force University animal experiment ethics committee.

\section{Animal grouping and establishment of the rat model of overactive bladder syndrome}

The investigators were blinded to group allocation during the experiments and analysis. The animals were fasted for $12 \mathrm{~h}$ before beginning the experiments. By random number table grouping method, the animals were randomly assigned to the sham intervention group, sham+saline group, sham+ZD7288 group, OAB group, $\mathrm{OAB}+$ saline group, and $\mathrm{OAB}+\mathrm{ZD} 7288$ group. The sample size calculation of each group was based upon our pilot study. In our pilot study, we used 3 animals in each of the six groups and totally 18 rats. And we got the preliminary data from the urodynamic studies from the six experimental groups. And mean \pm SD of Micturition Interval $(\mathrm{Sec})$ in the six groups were $65.83 \pm 11.27$, $65.42 \pm 10.42, \quad 67.22 \pm 14.16, \quad 45.89 \pm 9.76, \quad 42.35 \pm 9.54$, $54.28 \pm 6.60$ respectively. Method of one-way analysis of variance was used to estimate sample size. According to pilot study results, the means of six groups were set to $66,65,67,46,42,54$. And the standard deviation was preset to 14, because the largest SD was 14.16. Significance level was set to 0.05 for two-sided tests $(\alpha=0.05)$, and the power was set to $0.9(\beta=0.1)$. Estimation results showed that the sample size of each group should be no less than 7. In the present study, the real sample size of each group was from our actual experiments, and more than 7 animals were used in each group. We have not ruled out any raw data. Consequently, 8 to 11 animals from each group were used for this study, and the number of animals or DRG cells used were indicated in the tables and figure legends, respectively. Similar researches regarding sample size were reported previously $[10,11]$. An intraperitoneal injection comprising 1\% sodium pentobarbital $(50 \mathrm{mg} / \mathrm{kg}$ body weight, sigma, USA) was administered to anaesthetize the animals, A $1.1 \mathrm{~mm}$ 
diameter indwelling catheter (PE20, Smith Medical, England), lubricated with paraffin oil was gently inserted into the lightly pinched external urethral orifice, and passed through the urethra into the bladder. A $1.5 \mathrm{~cm}$ median incision was made in the lower abdomen. The neck of the bladder and the proximal urethra were bluntly separated using a sterilized cotton swab, and the rear of the bladder neck was bluntly separated exposing the bilateral ureters using an ophthalmic forceps in order to avoid ligation. The bladder was lifted, and the potential lacuna between the back of the urethra and the uterus was opened via blunt and sharp dissection, a 3-0 thread was inserted through it, and the proximal urethra ligated. The degree of tightness of the thread knot can be adjusted and it can be moved along with the surrounding tissue when pulling the indwelling catheter, so that the catheter can be easily removed. Four weeks after the initial surgery, the rats were anaesthetized using an intraperitoneal injection of $25 \%$ urethane $(1.0 \mathrm{mg} / \mathrm{kg})$. The lower abdomen was cut open and the pelvic cavity entered to expose the bladder. The upper part of the bladder was incised, and a plastic bushing inserted and fastened using purse string suture, after which a micro perfusion pump (Promed-Tech., Bellingham, MA, USA) and a three-way connection pressure sensing device (Nidoc970 urodynamic instrument, Yong xin, China) were connected to the other end of the bushing. The pressure measurement parameters were set to pressure mode, scanning speed of $10 \mathrm{~s} / \mathrm{div}$, direct current timeconstant, acquisition frequency of $400 \mathrm{~Hz}$, and filtering frequency of $30 \mathrm{~Hz}$. In order to calibrate the zero value, physiological saline at $37^{\circ} \mathrm{C}$ was administered into the bladder at $0.3 \mathrm{~mL} / \mathrm{min}$, with concomitant digital data collection. If during the low-pressure filling period the bladder contraction interval was shortened and the internal bladder pressure surpassed $15 \mathrm{~mm} \mathrm{H}_{2} \mathrm{O}$, the $\mathrm{OAB}$ animal model was considered successfully developed, and further experiments were conducted.

\section{Determination of urodynamic parameters}

In accordance with a published method [12], after having been prevented from drinking but not from eating the night before, the rats were anaesthetized by intraperitoneally administered $25 \%$ urethane $(1.0 \mathrm{~g} / \mathrm{kg})$, and a 24-gauge catheter (Surflo, Terumo Corp., Tokyo, Japan) was buried within the dome of the bladder. A threechannel connector was used to connect the catheter and the pressure sensor (TP-200 T, Nihon Kohden, Tokyo, Japan). The catheter was also connected via a threefold to a pump for continuous administration of physiological saline at $0.1 \mathrm{~mL} / \mathrm{min}$. Bladder pressure and urinary volume were recorded by the software on the connected computer during the saline administration process. The measured parameters also included micturition interval (MI), micturition volume (MV), micturition time (MT), pressure difference between the maximal micturition pressure and the basal pressure (MP-BP), and the pressure difference between the threshold pressure and basal pressure [13]. For each animal, 6-8 micturition cycles were recorded, and the average value was taken as the final result [14].

\section{Electrophysiological characterization of dorsal root ganglia (DRG) Preparation of DRG samples}

After anesthesia via intraperitoneal injection of sodium pentobarbital $(40 \mathrm{mg} / \mathrm{kg})$, the L6 DRG was exposed and completely separated from surrounding muscle tissue using a glass needle. The extracted DRG was placed into artificial cerebrospinal fluid (ACSF, comprising (mM): $\mathrm{NaCl} 124, \mathrm{MgCl}_{2} 1, \mathrm{KCl} 2.5, \mathrm{NaH}_{2} \mathrm{PO}_{4} 1.2, \mathrm{CaCl}_{2} 2$, $\mathrm{NaHCO}_{3}$ 25, Glucose 10, pH 7.4 set using $1 \mathrm{~N} \mathrm{HCl}$ or $\mathrm{NaOH}$, osmotic pressure set to 290-310 mOsm), which had been air-saturated for $30 \mathrm{~min}$. The upper surface of the specimen was cleaned from connective tissue and covering membranes under a dissecting microscope, after which it was placed into a centrifuge tube containing $0.5 \mathrm{~mL}$ of a protease solution comprising $1.0 \mathrm{mg} / \mathrm{mL}$ collagenase (Sigma, USA) and $0.4 \mathrm{mg} / \mathrm{mL}$ trypsin (Sigma, USA) in PBS $\mathrm{pH} 7.4$, and incubated in a water bath at $37^{\circ} \mathrm{C}$ for $40 \mathrm{~min}$. After digestion was completed, the DRG specimen was removed and washed three times with $25 \mathrm{~mL}$ ACSF, after which it was incubated in airsaturated ACSF for $1 \mathrm{~h} \mathrm{[15].}$

\section{Whole-cell patch-clamp measurements}

The DRG sample was carefully transferred to a perfusion chamber (SHD-27LPKIT, DL Nature gene Life Science, China), fastened using a $\mathrm{u}$-shaped platinum sample holder, and irrigated using a perfusion pump (BT100-2 J/DG-2, Longer, China) at a controlled rate of $1-2 \mathrm{~mL} /$ min. An upright microscope was used to investigate the intactness and distribution of cells in the DRG sample, and a cell with a clear $35-40 \mu \mathrm{m}$ outline and clean surface was selected as the patch-clamp target. A glass electrode (TW150F-4, WPI, USA, electrode resistance 3-6 $\mathrm{M} \Omega$ ) filled with the appropriate amount of electrode solution (mM: Glucose 120, $\mathrm{MgCl}_{2} 2, \mathrm{KCl} 18, \mathrm{CaCl}_{2} 1$, EGTA 5, HEPES 10, $\mathrm{Na}_{2}$-ATP 5, $\mathrm{Na}_{3}$-GTP 0.4, pH set to 7.4 using $1 \mathrm{~N} \mathrm{HCl}$, osmotic pressure set to $280-300$ mOsm) was placed into the electrode holder, after which a $1 \mathrm{~mL}$ syringe was used to apply positive pressure to prevent contamination of the electrode tip. As the electrode was slowly moved toward the target cell, an "umbilical" indentation formed. When the electrical resistance in this seal test rose to $0.1-0.3 \mathrm{M} \Omega$, the positive pressure was removed, providing a small amount of negative pressure, forming a high-resistance seal 
between the cell and the electrode tip. This ensured a $\mathrm{G} \Omega$ resistance level, which induced the cells to rupture the membrane, thus forming the whole-cell patch clamp. After the clamp was formed, $\mathrm{Cm}, \mathrm{Vm}$ and Ra were measured. Under the current clamp, the cell's resting potential was measured, and cells exhibiting a Ra value $<20$ $\mathrm{M} \Omega$ and a resting potential below $-50 \mathrm{mV}$ were selected for further experiments [15].

Under the electrical-current clamp, the DRG neuron was stimulated with a $600 \mathrm{~ms}$ depolarizing square wave based on the resting potential, at 100-pA intervals. The induced action potentials (AP) were recorded, and their characteristics analyzed. The first action potential was chosen to determine changes of the AP amplitude, AHP amplitude, threshold value and half width, as well as the membrane potential, $\mathrm{Cm}$ and $\mathrm{Rm}$. The amplitude of the AP $(\mathrm{mV})$ was defined as the difference in electric potential between its beginning and highest point, the threshold value $(\mathrm{mV})$ was defined as the membrane potential level at $30 \%$ of the rising slope after AP differentiation, the half width (ms) refers to the time difference between two corresponding points on the ascending and descending branches starting at the beginning of the AP, and AHP amplitude denotes the difference in electric potential between the membrane potential and the lowest point of the hyperpolarization. The threshold strength of the medium-size DRG cells (expressed in $\mathrm{pA}$ ), defined as the smallest current that can elicit an AP from the cells, was recorded under the current clamp. During the experiment, the cells were stimulated with a square-wave stimulus, starting from $-100 \mathrm{pA}$ and rising in steps of $10 \mathrm{pA}$, with a duration of $60 \mathrm{~ms}$. In order to prevent cell damage, the upper limit of the square-wave stimulus was set to $1000 \mathrm{pA}$. The 1.5-fold current strength of the rheobase was used to record the number of APs in the medium-size DRG cells, yielding the statistical current strength, with a square-wave stimulus duration of $600 \mathrm{~ms}$. The cells were clamped at $-60 \mathrm{mV}$, in order to stimulate an $I_{\mathrm{h}}$, and a hyperpolarization potential was applied starting from $-110 \mathrm{mV}$ and increasing in $10 \mathrm{mV}$ steps to $-60 \mathrm{mV}$. The slow depolarization constant $\tau$ was fitted from $I_{\mathrm{h}}$ using the formula $\mathrm{It}=I_{\mathrm{ss}}+$ $I_{\mathrm{h}}$ exp. $-\mathrm{t} / \mathrm{\tau}$, to obtain the size of the current for time $\mathrm{t}$, whereby $I_{\mathrm{ss}}$ is the steady-state current value [7].

\section{Intrathecal injection of ZD7288}

Intrathecal cathetererization was conducted according to a published report [16]. Briefly, an incision encompassing $1.5-2.0 \mathrm{~cm}$ was made along the spinous processes of the spine, and the muscle tissue separated via blunt dissection. A PE-10 catheter (Smith Medical, England), was slowly inserted with its port plugged into the intervertebral foramen, and fixed with dental cement once it came in contact with the DRG. The control group animals also had PE-10 catheters inserted into the intervertebral foramen and fixed with dental cement. A $1 \mathrm{~cm}$ segment of the fixed catheter was exposed. After burying the tube, the animals' locomotor function was investigated, and individuals with normal mobility were used for further experiments. Three days after surgery, ZD7288 (Sigma, USA) was injected into the exposed catheter ( $2 \mu \mathrm{M}$, in $10 \mu \mathrm{L}$ of physiological saline, once per day) and continued for 7 days. The control group was injected with the corresponding amount of pure physiological saline. After pharmaceutical administration for 7 days, the urodynamic parameters of the administered group were measured again. Animals were sacrificed via an overdose of sodium pentobarbital at the end of the experiment.

\section{Statistical analysis}

The experimental data are represented as mean \pm SD. The patch clamp data were analyzed using the Clampfit software (Version 9.2, Axon, USA), charts were drawn using Origin 8.0, and statistical analysis was conducted using SPSS 16.0 (SPSS, USA). Statistical analyses were performed by with Student's $t$ test when two groups were compared. Results for more than two groups were evaluated by one-way ANOVA followed by Tukey post hoc test. Results were considered statistically significant at $p<0.05$.

\section{Results \\ ZD7288 significantly reduces urinary bladder weight in $\mathrm{OAB}$ rats}

The average whole-body and bladder weights of the rats in each group are listed in Table 1 . The OAB rats did not show any statistically significant differences in body weight $(p>0.05)$. One week after the OAB rats were intrathecally administered either ZD7288 or saline at the L6 DRG, the body weight also did not change in a statistically significant way $(p>0.05)$. Furthermore, following the experimental endpoint, the urinary bladders of the SD rats were removed, and their weights were found to be significantly increased in the treatment group compared to the sham- group $(p<0.01$,$) . Furthermore, this$ increase was significantly lowered upon ZD7288 administration ( $p<0.01$ compared to the sham- group). Intrathecal administration of saline did not have a significant effect on the bladder weight of the OAB rats $(p>0.05$, $\mathrm{OAB}$ vs $\mathrm{OAB}+$ saline).

\section{Decrease of urinary bladder function in the $O A B$ rats}

Table 2 lists the micturition time, micturition interval, micturition volume, the difference between maximal micturition pressure and the basal pressure, as well as the difference between the threshold pressure and basal pressure. Statistical analysis revealed that in the $\mathrm{OAB}$ 
Table 1 Average whole-body and bladder weights of the rats in different groups after intrathecal pharmaceutical administration for 7 days

\begin{tabular}{lllll}
\hline Parameter & Sham intervention & OAB & OAB + saline & OAB + ZD7288 \\
\hline $\begin{array}{l}\text { Body weight } \\
\text { (g) }\end{array}$ & $233.21 \pm 11.12$ & $229.34 \pm 12.13$ & $235 \pm 10.67$ & $231.41 \pm 13.51$ \\
Bladder weight $(\mathrm{mg})$ & $121.32 \pm 7.56$ & $214.53 \pm 11.42^{* *}$ & $211.34 \pm 12.28$ & $187.38 \pm 12.63^{\# \#}$ \\
$\mathrm{n}$ & 8 & 11 & 11 & 11 \\
\hline
\end{tabular}

$O A B$ Overactive bladder syndrome group; $O A B+$ saline: $O A B$ group treated with saline; $O A B+Z D 7288$ : OAB group treated with $Z D 7288$; ${ }^{*} p<0.01$, comparison between the $O A B$ and sham-intervention groups; ${ }^{\# \#} p<0.01$, comparison between the OAB and OAB + ZD7288 groups (one-way ANOVA)

group, the micturition interval was significantly shortened $(p<0.01)$, micturition volume was significantly decreased $(p<0.01)$, and the duration of micturition was significantly longer $(p<0.05)$, compared to the sham group. The difference between the basal and maximal urination pressure, as well as the difference between the threshold pressure and the basic pressure did not change significantly $(p>0.05$, OAB vs sham). One week after intrathecal administration of ZD7288, the micturition interval and volume of OAB + ZD7288 rats were significantly increased, compared with $\mathrm{OAB}$ rats $(p<0.01)$. The micturition time was also significantly reduced $(p<0.05, \mathrm{OAB}+\mathrm{ZD} 7288$ vs OAB $)$. However, $2 \mu \mathrm{M}$ ZD7288 did not significantly affect either the difference between the basal and maximal urine pressure, or the difference between the basal and the threshold pressure $(p>0.05, \mathrm{OAB}+\mathrm{ZD} 7288$ vs OAB). We also investigated the effects of intrathecal administration of saline on voiding function, without discovering significant differences in any of the experimentally determined parameters $(p>0.05$, OAB vs OAB + saline $)$.

\section{Increase of excitability of the medium-size DRG neurons in the $O A B$ rats}

In the whole-call patch clam experiment, we recorded the resting membrane potential (RMP), cell capacitance $(\mathrm{Cm})$, and input resistance $\left(\mathrm{R}_{\mathrm{in}}\right)$ of the medium-size DRG neurons. As is shown in Table 3, the RMP and $R_{\text {in }}$ of the OAB rats were significantly decreased $(p<0.05)$, while the cells' radius and $\mathrm{Cm}$ did not exhibit significant changes compared with the sham group $(p>0.05)$.
Thus, the passive membrane characteristics of the medium-size DRG cells did not change significantly under $\mathrm{OAB}$ conditions. However, did the active characteristics change? Compared with the sham group, the AP amplitude of the medium-size DRG neurons in the OAB group was significantly increased (from $95.64 \pm 5.70 \mathrm{mV}$ to $110.21 \pm 12.34 \mathrm{mV}$ ), and the halfwidth decreased from $1.67 \pm 0.45 \mathrm{~ms}$ to $1.24 \pm 0.32 \mathrm{~ms}$ (Fig. 1c), the afterhyperpolarization amplitude decreased from $12.45 \pm 1.45 \mathrm{mV}$ to $10.37 \pm 1.37 \mathrm{mV}$ (Fig. 1c), and the threshold value was significantly less negative $(-31.03 \pm 3.24 \mathrm{mV}$ compared to $-38.10 \pm$ $2.31 \mathrm{mV}$ in the control, Fig. 1d) with $p$ values $<0.05$ in all cases (OAB vs sham).Taken together, these results indicate that the excitability of the medium-size DRG neurons of the OAB rats was significantly increased. Furthermore, we investigated the lowest stimulus strength of the current (equal to the rheobase) capable of eliciting an AP from the mediumsize DRG neurons. The results show that the rheobase was significantly decreased in the $\mathrm{OAB}$ group $(365.36 \pm 10.54 \mathrm{pA}$ in sham vs $234.67 \pm 17.45 \mathrm{pA}$ in $\mathrm{OAB}, p<0.05$, Fig. 2a).

In order to quantify the cells' excitability, we measured the number of action potentials (APs) induced by a current corresponding to the 1.5-fold of the rheobase. The results thus represent the number of APs induced by a current of $500 \mathrm{pA}$, and the number of APs induced in cells from the $\mathrm{OAB}$ group was significantly higher than in the sham group $(14.63 \pm 4.35$ in OAB vs $2.42 \pm$ 0.51 in sham, $p<0.01$, Fig. $2 c$ and d).

Table 2 Urinary bladder and voiding parameters, determined for each group of animals after intrathecal pharmaceutical administration for 7 days

\begin{tabular}{|c|c|c|c|c|}
\hline Parameter & Sham intervention & $O A B$ & $\mathrm{OAB}+$ saline & $\mathrm{OAB}+\mathrm{ZD} 7288$ \\
\hline Micturition Interval (Sec) & $75.23 \pm 5.71$ & $32.85 \pm 4.32^{* *}$ & $33.45 \pm 3.78$ & $48.18 \pm 6.58^{\# \#}$ \\
\hline Micturition Volume (ml) & $0.63 \pm 0.42$ & $0.21 \pm 0.05^{* *}$ & $0.34 \pm 0.24$ & $0.58 \pm 0.32^{\# \#}$ \\
\hline Micturition Time (s) & $10.38 \pm 0.74$ & $12.65 \pm 1.31^{*}$ & $13.02 \pm 1.54$ & $10.54 \pm 1.53^{\#}$ \\
\hline Difference between the Maximal and Basal Micturition Pressures $\left(\mathrm{cmH}_{2} \mathrm{O}\right)$ & $54.35 \pm 4.67$ & $52.28 \pm 7.49$ & $51.76 \pm 6.98$ & $49.53 \pm 4.87$ \\
\hline Difference between the Threshold Pressure and Basal Pressure $\left(\mathrm{cmH}_{2} \mathrm{O}\right)$ & $12.86 \pm 1.08$ & $9.59 \pm 6.75$ & $9.78 \pm 7.31$ & $8.87 \pm 7.21$ \\
\hline $\mathrm{n}$ & 8 & 11 & 11 & 11 \\
\hline
\end{tabular}


Table 3 Passive electrophysiological characteristics of the middle-size DRG neurons in the OAB and sham-intervention groups

\begin{tabular}{lll}
\hline Parameter & Sham intervention & OAB \\
\hline Cell size $(\mu \mathrm{m})$ & $41.36 \pm 1.34$ & $42.21 \pm 1.25$ \\
$\mathrm{RMP}(\mathrm{mV})$ & $-58.49 \pm 1.72$ & $-51.03 \pm 2.45^{*}$ \\
$\mathrm{R}_{\text {in }}(\mathrm{M} \Omega)$ & $54.87 \pm 5.36$ & $49.38 \pm .54^{*}$ \\
$C_{\mathrm{m}}(\mathrm{pF})$ & $63.41 \pm 6.37$ & $65.39 \pm 7.82$ \\
$\mathrm{n}$ & 24 & 19 \\
\hline
\end{tabular}

$O A B$ denotes the overactive bladder syndrome group, $\mathrm{n}$ represents the number of individual investigated cells, $R M P$ is the resting membrane potential, $R_{\text {in }}$ is input resistance, and $C_{m}$ is cell capacitance. Comparison of DRG in the $O A B$ and sham-intervention groups revealed no significant difference in $C_{m}(p>0.05$, Student's $t$ test), while the RMP was significantly less negative and $R_{\text {in }}$ was significantly decreased in the OAB group ${ }^{*} p<0.05$ for both, Student's $t$ test)

\section{ZD7288 significantly decreased the excitability of the medium-size DRG cells in the $O A B$ rats}

Taken together, the above-stated results indicate that the excitability of the medium-size DRG cells increased in the $\mathrm{OAB}$ rats. Our research further revealed that an intrathecal injection of ZD7288 was able to significantly decrease the $O A B$ rats' bladder weight as well as to significantly improve their voiding function (Table 2). This in turn means that ZD7288 can influence the excitability of the DRG neurons, reducing the DRG-mediated activation of the bladder, and thus lowering bladder motility. The $I_{\mathrm{h}}$ current induces the slow depolarization of the cell membrane at the beginning of the AP, so that, after the AP burst and until the hyperpolarization potential, there is an inactivation of the $I_{\mathrm{h}}$ load-bearing channel. Therefore, the $I_{\mathrm{h}}$ current can influence the AP threshold

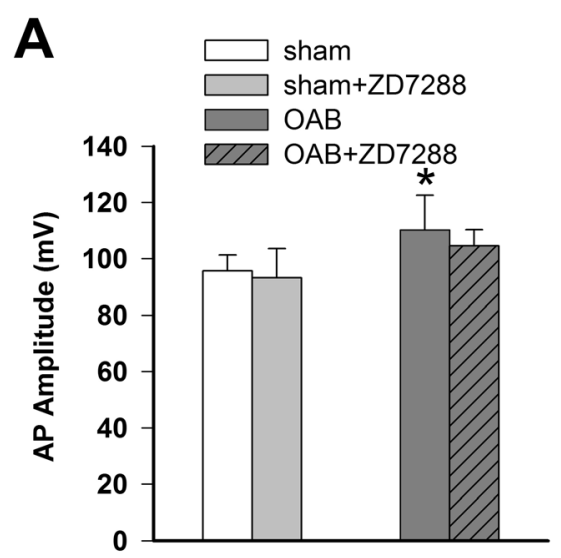

B
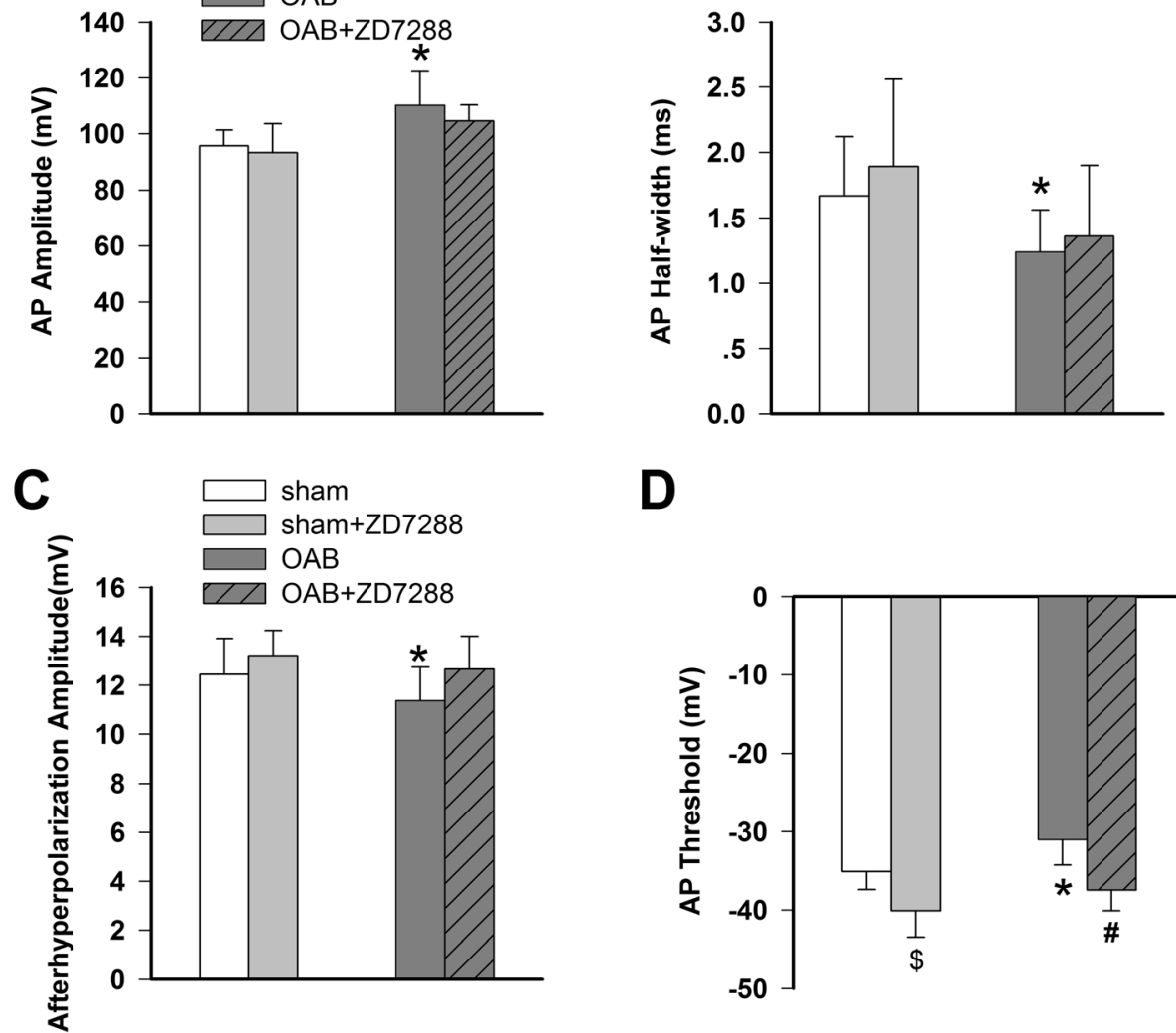

D

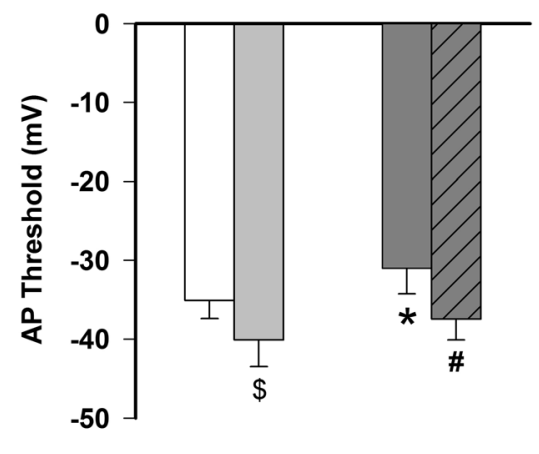

Fig. 1 Effects of ZD288 on the AP amplitude, half-width, threshold value, and after hyperpolarization amplitude of the medium-size DRG neurons in OAB and sham-intervention rats. The amplitude, half-width, afterhyperpolarization potential (AHP) amplitude and threshold value of the first induced action potential (AP) of the medium-size DRG cells in the OAB rat model were investigated, together with the effect of ZD7288 ( $2 \mu M)$ administration on said parameters. $\mathbf{a}$ : the amplitude of the action potential was significantly increased in OAB rats, $\mathbf{b}$ : the action potential halfwidth was significantly reduced in $O A B$ rats, $\mathbf{c}$ : the amplitude of the afterhyperpolarization potential was significantly reduced in $O A B$ rats, $\mathbf{d}$ : the action potential threshold value of OAB rats was significantly lowered; ZD7288 significantly reduced the threshold value of both OAB and shamintervention rats, but did not affect the AP amplitude, half-width, and AHP amplitude. ( ${ }^{*} p<0.05$, sham vs OAB group, ${ }^{*} p<0.05, O A B$ vs $O A B+$ ZD7288 group; ${ }^{\$} p<0.05$, sham vs sham+ZD7288 group; sham $n=24$, sham+ZD7288 $n=24, \mathrm{OAB} n=19$, OAB +ZD7288 $n=19$ 


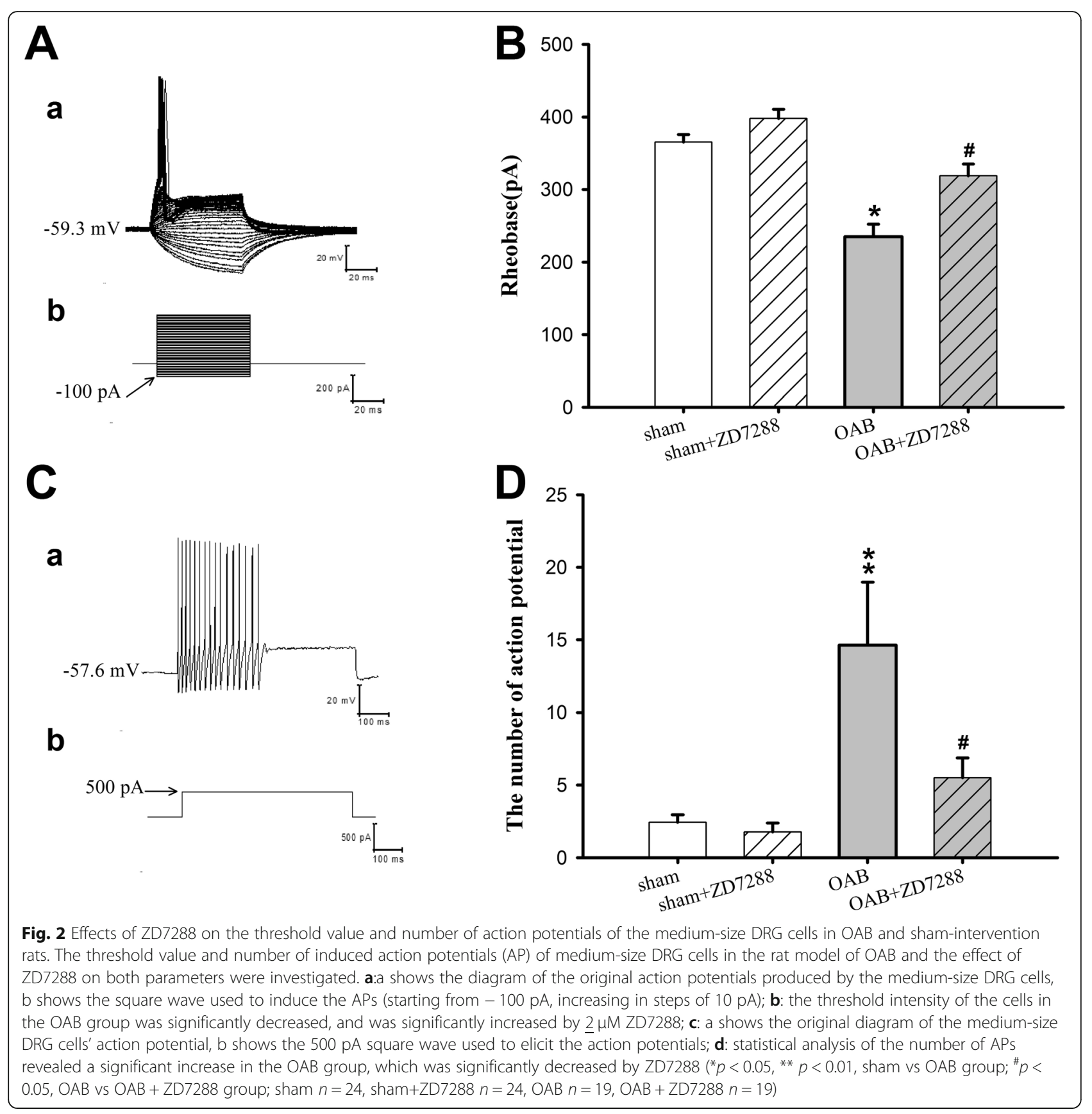

value, which is consistent with the finding that the threshold value was significantly increased by ZD7288 in both the $\mathrm{OAB}$ and sham groups $(-35.03 \pm 2.31 \mathrm{Mv}$ in sham vs $-40.10 \pm 3.41 \mathrm{mV}$ in sham+ZD7288; $-31.03 \pm$ $3.24 \mathrm{Mv}$ in $\mathrm{OAB}$ vs $-37.46 \pm 2.66 \mathrm{mV}$ in $\mathrm{OAB}+\mathrm{ZD} 7288$, $p<0.05$ in both cases; Fig. 1d). The rheobase of the medium-size cells in the L6 DRG ganglia of the OAB animals was also significantly increased by ZD7288 $(234.67 \pm 17.45 \mathrm{pA}$ in $\mathrm{OAB}$ vs $318.82 \pm 16.39 \mathrm{pA}$ in $\mathrm{OAB}+\mathrm{ZD} 7288, p<0.05$; Fig. 2b). Additionally, the experiments revealed that the number of APs induced by a
500-pA current was significantly decreased in the $O A B+$ ZD7288 group $(14.63 \pm 4.35$ in $\mathrm{OAB}$ vs $5.50 \pm 1.37$ in $\mathrm{OAB}+\mathrm{ZD} 7288, p<0.05$; Fig. 2d).

However, ZD7288 did not have any significant effect on the amplitude of the afterhyperpolarization potential or half-width $(p>0.05, \mathrm{OAB}+\mathrm{ZD} 7288$ vs $\mathrm{OAB}$ and sham + ZD7288 vs sham; Figs. 1a, b and c).

During the AP hyperpolarization process, the $I_{\mathrm{h}}$ current is activated and involves in the recovery of the membrane following the AHP $[17,18]$. This means that the $I_{\mathrm{h}}$ current may possibly influence the time-course of 


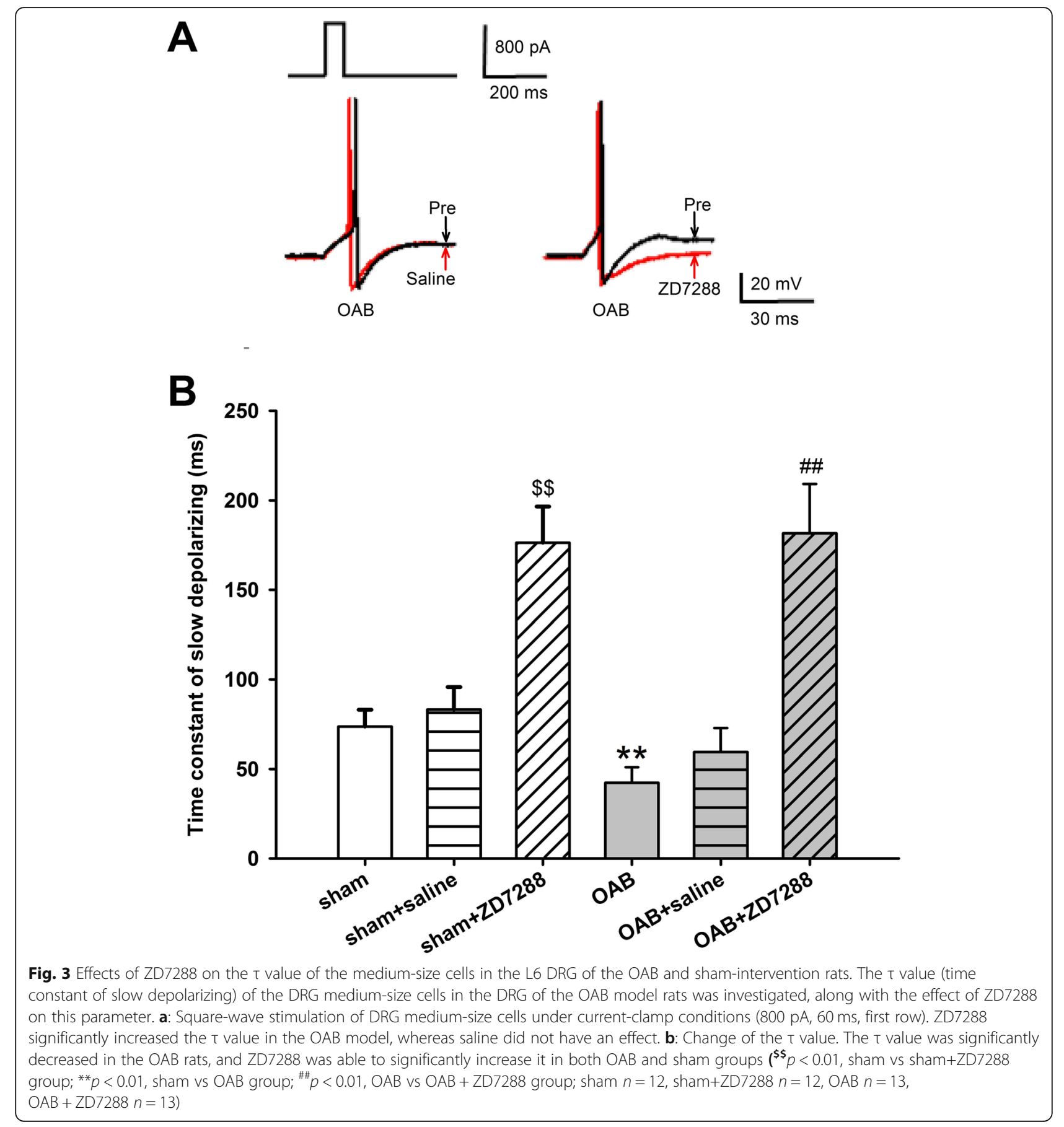

the recovery of the hyperpolarization potential towards the level of the membrane potential. The experiments have uncovered that the $\tau$ value in the $O A B$ model was significantly reduced $(73.7 \pm 9.4 \mathrm{~ms}$ in sham vs $42.35 \pm$ $8.67 \mathrm{~ms}$ in OAB; $p<0.01$; Fig. $3 \mathrm{~b}$ ), indicating that the excitability of the OAB rats' medium-size DRG cells increased, which was in agreement with the increase of the number of preceding discharges. ZD7288 $(2 \mu \mathrm{M})$ was able to significantly increase the $\tau$ values of both the
$\mathrm{OAB}$ and sham animals $(73.7 \pm 9.4 \mathrm{~ms}$ in sham vs $176.34 \pm 20.30 \mathrm{~ms}$ in sham+ZD7288; $42.35 \pm 8.67 \mathrm{~ms}$ in $\mathrm{OAB}$ vs $181.56 \pm 27.45 \mathrm{~ms}$ in $\mathrm{OAB}+\mathrm{ZD} 7288 ; p<0.01$, in both cases; Fig. 3b).

At the same time, saline administration did not have a significant effect on the $\tau$ value of either the $\mathrm{OAB}$ or the sham group $(73.7 \pm 9.4 \mathrm{~ms}$ in sham vs $83.26 \pm 12.56 \mathrm{~ms}$ in sham+saline; $42.35 \pm 8.67 \mathrm{~ms}$ in $\mathrm{OAB}$ vs $59.44 \pm 13.43$ $\mathrm{ms}$ in $\mathrm{OAB}+$ saline; $p>0.05$ in both cases; Fig. $3 \mathrm{~b}$ ). 
Measurement of $I_{\mathrm{h}}$ current density in the medium-size DRG cells of the OAB rats with ZD7288 treatment

The above-described experiments revealed that ZD7288 is able to significantly reduce the threshold value, threshold intensity, and number of APs, in addition to significantly increasing the $\tau$ value of the L6 DRG neurons in the $\mathrm{OAB}$ rats. These results thus indicate that the formation of the $I_{\mathrm{h}}$ current in the OAB model might play an inordinately important role. In the voltage clamp experiments, the cells were clamped at $-60 \mathrm{mV}$, and the hyperpolarization potential was increased in $10 \mathrm{mV}$ steps from $-110 \mathrm{mV}$ to $-60 \mathrm{mV}$, inducing a slow inward cation current (Fig. 4a). Furthermore, this type of hyperpolarization-activating inward current could be completely blocked by $2 \mu \mathrm{M}$ ZD7288, confirming that the recorded current is indeed the $I_{\mathrm{h}}$ current. When the potential was clamped at $-90,-100$ and $-110 \mathrm{mV}$, the $I_{\mathrm{h}}$ current density was significantly higher in $\mathrm{OAB}$ group compared with control (at $-90 \mathrm{mV}: 2.68 \pm 1.67 \mathrm{pA} / \mathrm{pF}$ in sham vs $8.43 \pm 2.10 \mathrm{pA} / \mathrm{pF}$ in $\mathrm{OAB}$; at $-100 \mathrm{mV}: 4.56 \pm$ $1.79 \mathrm{pA} / \mathrm{pF}$ in sham vs $9.73 \pm 2.67 \mathrm{pA} / \mathrm{pF}$ in $\mathrm{OAB}$; at $110 \mathrm{mV}: 6.12 \pm 2.13 \mathrm{pA} / \mathrm{pF}$ in sham vs $14.39 \pm 2.87 \mathrm{pA} /$ $\mathrm{pF}$ in $\mathrm{OAB} ; p<0.05$, Fig. $4 \mathrm{~b})$. In contrast, $15 \mathrm{~min}$ after $2 \mu \mathrm{M}$ ZD7288 was added to the measurement buffer in

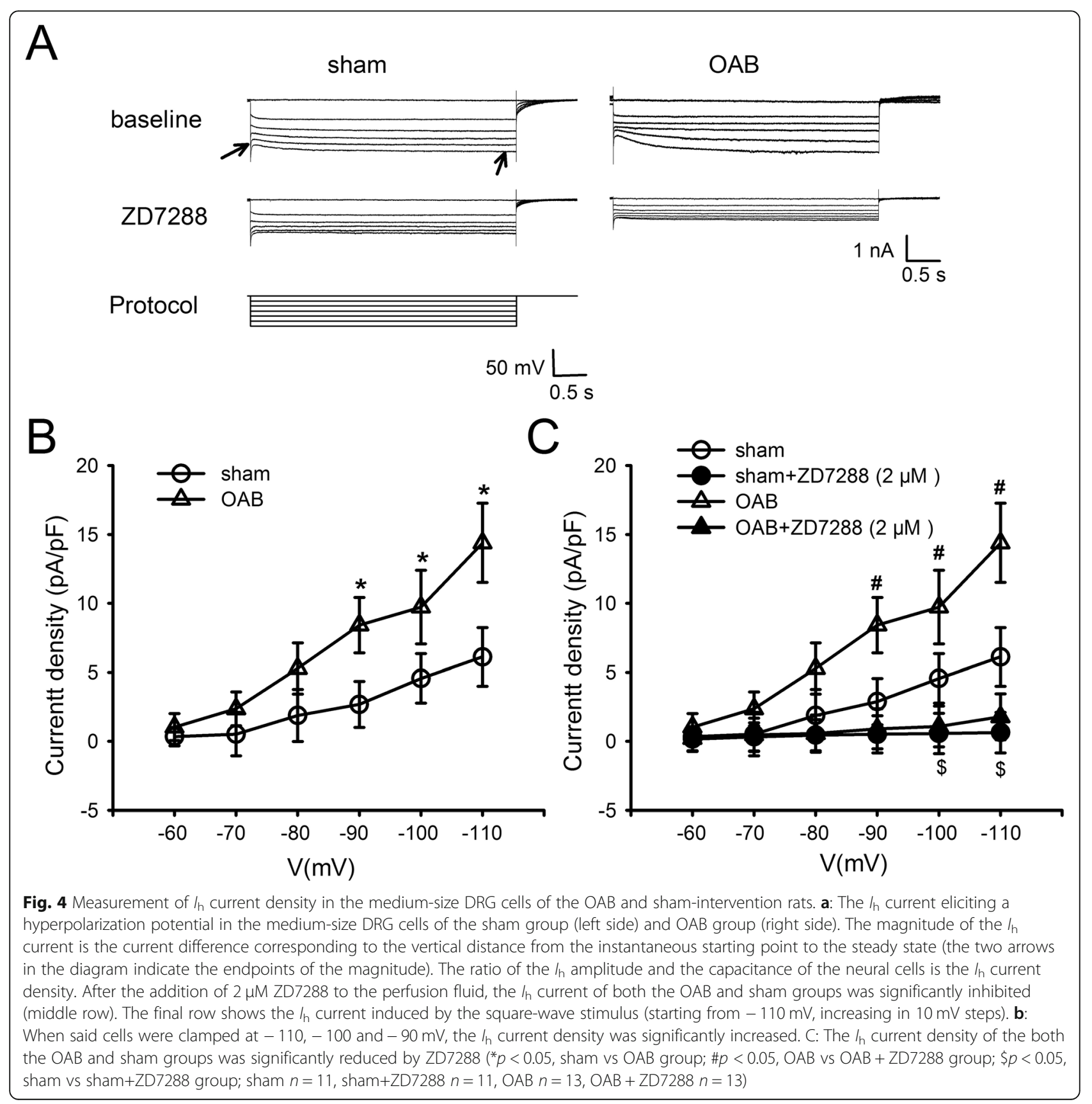


which the cells were immersed, the $I_{\mathrm{h}}$ current density of the DRG neurons in the OAB group was significantly lower (at $-90 \mathrm{mV}, 8.43 \pm 2.10 \mathrm{pA} / \mathrm{pF}$ in $\mathrm{OAB}$ vs $0.91 \pm$ $1.45 \mathrm{pA} / \mathrm{pF}$ in $\mathrm{OAB}+\mathrm{ZD} 7288$; at $-100 \mathrm{mV}, 9.73 \pm 2.67$ $\mathrm{pA} / \mathrm{pF}$ in $\mathrm{OAB}$ vs $1.08 \pm 1.48 \mathrm{pA} / \mathrm{pF}$ in $\mathrm{OAB}+\mathrm{ZD} 7288$; at $-110 \mathrm{mV}, 14.39 \pm 2.87 \mathrm{pA} / \mathrm{pF}$ in $\mathrm{OAB}$ vs $1.78 \pm 1.67$ $\mathrm{pA} / \mathrm{pF}$ in $\mathrm{OAB}+\mathrm{ZD} 7288 ; p<0.05$, Fig. 4c).

During the same experiment, it was discovered that ZD7288 was able to significantly reduce the $I_{\mathrm{h}}$ current density of the DRG neurons in the sham rats (at -100 $\mathrm{mV}: 4.56 \pm 1.79 \mathrm{pA} / \mathrm{pF}$ in sham vs $0.57 \pm 1.46 \mathrm{pA} / \mathrm{pF}$ in sham+ZD7288; at $-110 \mathrm{mV}: 6.12 \pm 2.13 \mathrm{pA} / \mathrm{pF}$ in sham vs $0.64 \pm 1.47 \mathrm{pA} / \mathrm{pF}$ in sham+ZD7288; $p<0.05$; Fig. $4 \mathrm{c}$ ). However, the reduction range of current density of DRG neurons after ZD7288 administration were obviously wider in $\mathrm{OAB}$ rats than sham rats (Fig. 4c). These results suggested that ZD7288 could inhibit cell excitability of DRG cells relatively more strongly in OAB group than in sham group.

\section{Discussion}

The urinary bladder weight of the overactive bladder syndrome $(\mathrm{OAB})$ model rats, as well as the excitability and $I_{\mathrm{h}}$ current density of the animals' medium-size DRG neurons were significantly increased. Intrathecal injection of the specific $I_{\mathrm{h}}$ current inhibitor ZD7288 was able to alleviate the observed changes in the $\mathrm{OAB}$ rats, including a prolonged micturition time and shortened micturition interval. At the same time, ZD7288 completely blocked the $I_{\mathrm{h}}$ current density, and lowered the excitability of the medium-size DRG cells. Taken together, these results strongly suggest that the $I_{\mathrm{h}}$ current may be a factor in the development of OAB.

The partial bladder outlet obstruction rat model is a good approximation of the condition seen in clinical patients with urinary bladder overactivity and urethral blockage syndromes, including a high degree of agreement in the observed pathological changes [19]. Therefore, the partial bladder outlet obstruction rat model was used in this study, and the urinary bladder weight of the successfully induced $\mathrm{OAB}$ rats was significantly increased, in agreement with the literature [13]. Recordings of the micturition process have uncovered that the micturition interval of the $\mathrm{OAB}$ rats was significantly shortened, their micturition time was significantly increased, and micturition volume was significantly decreased, indicating that the rats with the partiallyblocked urethra indeed had an abnormally enhanced micturition reflex.

According to anatomical studies, the neural fibers that innervate the bladder include the myelinated $\mathrm{A}_{\delta}$ and the myelin-free C-type fibers $[6,19,20]$. One part of these afferent fibers transverses the pelvis, arriving at the L6 and S1 DRG of the spine, and takes part in the initial stage of the micturition signal, while another part transverses the lower abdomen, arriving at the L1 and L2 level of the spine $[3,21,22]$. The DRG neurons are classified into small, medium and large types according to their cross-sectional diameter, and further separated according to the $A_{\alpha / \beta}, A_{\delta}$ and $C$ typology $[4,5]$. A subset of the nerve fibers that control the micturition process terminate at the L6 DRG, making the question pertinent whether the excitability of the OAB rats' L6 DRG has exhibited any changes. The results of present study indicated that the AP amplitude and number of APs elicited by a 500-pA stimulus were significantly increased, whereas the half-width, threshold strength, threshold value and the afterhyperpolarization potential, as well as the input resistance were significantly decreased. The membrane potential also had an obvious depolarization tendency. All these results suggested that the mediumsize L6 DRG cells of the OAB rats had a significantly increased excitability. The membrane potential of the L6 DRG neurons of the OAB rats also displayed obvious depolarization, with an increase in the number of APs elicited by a $500-$ pA stimulus, as well as a decrease of the input resistance and $\tau$ value. Thus, the hyperpolarization-induced cation current $\left(I_{\mathrm{h}}\right)$ might play an important role in the observed increased excitability of the medium-size neurons in the L6 DRG of the OAB rats. The $I_{\mathrm{h}}$ current is present in excitable cells in both the center and the periphery of the body, including the photoreceptor cells of the retina and the cardiac pacemaker cells, as well as cells of the central and peripheral nervous system $[23,24]$. The $I_{\mathrm{h}}$ current channel is activated when the AHP is rapidly increasing, and the influx of $\mathrm{Na}^{+}$causes a slow depolarization of the cell membrane. In peripheral nerves, $I_{\mathrm{h}}$ is found in the sensory DRG neurons [25]. Within these sensory neurons, it participates in the AHP process, and induces the cell membrane to recover to the resting potential after depolarization, shortening the AHP amplitude and duration $[26,17,27]$. Studies have also reported that the $I_{\mathrm{h}}$ current plays a role in the maintenance of membrane potential, influencing the cells' spontaneous and repeated discharge, as well as related phenomena [28, 29]. In our experiments, it was shown that the specific $I_{\mathrm{h}}$ current inhibitor ZD7288 is able to clearly inhibit the excitability of the medium-size DRG cells in the OAB rats, including a reduction of the number of APs elicited by a 500-pA stimulus and an increase of the $\tau$ value, indicating that the $I_{\mathrm{h}}$ current within the L6 DRG neurons plays an important role in the etiology of OAB. After administration of ZD7288, DRG in sham+ZD7288 group also showed elevated $\tau$ value and reduction in current density, suggested that $I_{\mathrm{h}}$ current inhibitor could reduce the cell membrane excitability in sham rats. However, the reduction range of current density of DRG neurons 
after ZD7288 administration were obviously wider in $\mathrm{OAB}+$ ZD7288 group than sham + ZD7288 group. These results suggested that ZD7288 could inhibit cell excitability of DRG cells relatively more strongly in OAB rats than in sham rats.

A study by Masuda and coworkers has found that the $I_{\mathrm{h}}$ current is primarily present in the medium-size DRG neurons that control the urinary bladder, and that inhibition of the current significantly increases the time from the highest AHP point until the return to the resting potential, and reduces the excitability of the medium-size DRG neurons [7]. These results imply that the excitability of the medium-size mechanical nociceptor cells in the DRG that control the urinary bladder may be influenced by the $I_{\mathrm{h}}$ current. This raises the question whether the $I_{\mathrm{h}}$ current in the medium-size DRG cells of the OAB mice experienced changes. Our results show that the $I_{\mathrm{h}}$ current density was significantly increased, and functional experiments have shown that intrathecal injection of ZD7288 significantly improved the micturition parameters of the $\mathrm{OAB}$ rats. The experimental results indicate that an increase in the strength of the $I_{\mathrm{h}}$ current in the medium-size cells of the L6 DRG might be an important component of the $\mathrm{OAB}$ development process. Furthermore, the hyperpolarization-activated cyclic nucleotide-gated cation channel $(\mathrm{HCN})$ mediates the $I_{\mathrm{h}}$ current, and an investigation of $\mathrm{HCN}$ expression in the neurons that control the bladder, which are found in the L6-S1 DRG, has uncovered rich expression of HCN2 in the average medium-size DRG cells.

It is thus theoretically possible that the significant increase of the $I_{\mathrm{h}}$ current in the medium-size neurons of the L6 DRG of OAB rats is related to the HCN2 channel, and this notion merits further research. Furthermore, these experimental results demonstrate that the half-width, amplitude, and afterhyperpolarization potential of the action potential are not influenced by ZD7288, which is in agreement with other studies, which have also found that ZD2788 cannot influence the shape of the AP or the AHP amplitude [28, 29]. This indicates that the observed changes of the $I_{\mathrm{h}}$ current may be part of the electrophysiological mechanism responsible for the increase of excitability in the medium-size DRG cells of the OAB rats. The limitations of current study is that the precise functional activity of the $I_{\mathrm{h}}$ current in the medium-size DRG neurons of OAB rats has not been fully unveiled, and we do not observe the small-size DRG neurons. Further studies using structurally dissimilar HCN channel blockers such as ivabradine will be needed to strengthen our present behavioral and electrophysiological findings. Further electrophysiological mechanisms influencing the cells' excitability still remain to be resolved. Since the etiology of OAB is complicated and multifactorial, additional studies are needed in different animal models and different $\mathrm{OAB}$ related cells to clarify the real role of $I_{\mathrm{h}}$ current in bladder dysfunction.

\section{Conclusions}

This study reveals that the excitability of medium-size cells in the L6 DRG of OAB model rats was significantly increased, and that the $I_{\mathrm{h}}$ current correlates with a part of the parameters of this heightened nerve-cell excitability. The medium-size cells in the L6 DRG of the OAB rats had a significantly increased $I_{\mathrm{h}}$ current density, and their micturition reflex was significantly improved under the influence of ZD7288, which indicates that the $I_{\mathrm{h}}$ current in these cells may be an important part of the electrophysiological mechanism leading to the development of OAB.

\section{Abbreviations \\ OAB: OVERACTIVE bladder syndrome; DRG: Dorsal root ganglia; MI: Micturition interval;; MV: Micturition volume; MT: Micturition time; MP- BP: Maximal micturition pressure and the basal pressure: \\ HCN: Hyperpolarization-activated cyclic nucleotide-gated cation; RMP: Resting membrane potential; AP: Action potentials; RMP: Resting membrane potential; Cm: Cell capacitance}

\section{Acknowledgements}

Not applicable.

\section{Authors' contributions}

C.T., F.Y., L.Y., and J.X. conceived and designed the experiments; W.Q., K.Z., and G.W. performed the experiments; C.T., F.Y., L.Y., and J.X. analyzed the data; F.L. provided all the funding, direction of the detail of experiments and design of the research. F.L. and J.Y. contributed reagents/materials/analysis tools. J.Y. has also designed the work and contributed to the data interpretation. C.T., F.Y., L.Y., and J.X. wrote the manuscript. All authors read and approved the final manuscript.

\section{Funding}

This work was supported by grants from the National Natural Science Foundation of China (No.81270844/30801142/81371615), Discipline promotion program of Xijing Hospital (XJZT19Z30) and from the Shaanxi Technology Research and Development Program (No.S2016SF-161/2013 K1205-11). The funding body did not have any role in the design of the study, the collection, analysis and interpretation of the data or the writing of the manuscript.

\section{Availability of data and materials}

The datasets used and/or analyzed during the current study are available from the corresponding author on reasonable request.

\section{Ethics approval and consent to participate}

This study was approved by the Ethics Committee of Xijing Hospital of Air Force Medical University.

\section{Consent for publication}

Not applicable.

\section{Competing interests}

The authors declare that they have no competing interests.

\section{Author details}

'Department of Urology, Xijing Hospital, Air Force Medical University, 15 Changle West Road, Xi'an 710032, Shaanxi, China. ${ }^{2}$ Xijing Hospital of Digestive Diseases, Xijing Hospital, Air Force Medical University, Xi'an, Shaanxi, China. ${ }^{3}$ Institute of neuroscience, Air Force Medical University, Xi'an, Shaanxi, China. 
Received: 29 January 2019 Accepted: 18 August 2020

Published online: 02 September 2020

\section{References}

1. Lee U, Scott V, Rashid R, Behniwal A, Wein A, Maliski S, Anger J. Defining and managing overactive bladder: disagreement among the experts. Urology. 2013;81(2):257-62.

2. Leong R, De Wachter S, van Kerrebroeck P. Current information on sacral neuromodulation and botulinum toxin treatment for refractory idiopathic overactive bladder syndrome: a review. Urol Int. 2010;84(3):245-53.

3. de Groat W, Yoshimura N. Anatomy and physiology of the lower urinary tract. Handb Clin Neurol. 2015;130:61-108.

4. Light A, Perl E. Spinal termination of functionally identified primary afferent neurons with slowly conducting myelinated fibers. J Comp Neurol. 1979; 186(2):133-50.

5. Réthelyi M. Preterminal and terminal axon arborizations in the substantia gelatinosa of cat's spinal cord. J Comp Neurol. 1977;172(3):511-21.

6. de Groat W, Nadelhaft I, Milne R, Booth A, Morgan C, Thor K. Organization of the sacral parasympathetic reflex pathways to the urinary bladder and large intestine. J Auton Nerv Syst. 1981;3(2-4):135-60.

7. Masuda N, Hayashi Y, Matsuyoshi H, Chancellor M, de Groat W, Yoshimura N. Characterization of hyperpolarization-activated current (Ih) in dorsal root ganglion neurons innervating rat urinary bladder. Brain Res. 2006;1096(1): 40-52.

8. Beaumont V, Zucker R. Enhancement of synaptic transmission by cyclic AMP modulation of presynaptic Ih channels. Nat Neurosci. 2000;3(2):133-41.

9. Chevaleyre V, Castillo P. Assessing the role of Ih channels in synaptic transmission and mossy fiber LTP. Proc Natl Acad Sci U S A. 2002:99(14): 9538-43.

10. Yoshimura N, Bennett NE, Hayashi Y, Ogawa T, Nishizawa O, Chancellor MB, de Groat WC, et al. Bladder overactivity and hyperexcitability of bladder afferent neurons after intrathecal delivery of nerve growth factor in rats. J Neurosci. 2006;26(42):10847-55.

11. Zotova EG, Christ GJ, Zhao W, Tar M, Kuppam SD, Arezzo JC. Effects of fidarestat, an aldose reductase inhibitor, on nerve conduction velocity and bladder function in streptozotocin-treated female rats. J Diabetes Complicat. 2007;21(3):187-95.

12. Liu HP, Chen GL, Liu P, Xu XP. Amlodipine alone or combined with terazosin improves lower urinary tract disorder in rat models of benign prostatic hyperplasia or detrusor instability: focus on detrusor overactivity. BJU Int. 2009:104(11):1752-7.

13. Jang H, Dong SH, Yuk SM. Changes of neuregulin-1(NRG-1) expression in a rat model of overactive bladder induced by partial urethral obstruction: is NRG-1 a new biomarker of overactive bladder? BMC Urol. 2013;13(1):54.

14. Liu F, Yao L, Yuan J, Liu H, Yang X, Qin W, Wu G, et al. Protective effects of inosine on urinary bladder function in rats with partial bladder outlet obstruction. Urology. 2009;73(6):1417.

15. Zhang M, Han W, Zheng J, Meng F, Jiao X, Hu S, Xu H. Inhibition of hyperpolarization-activated Cation current in medium-sized DRG neurons contributed to the Antiallodynic effect of Methylcobalamin in the rat of a chronic compression of the DRG. Neural Plast. 2015;2015:197392.

16. Yaksh TL, Rudy TA. Chronic catheterization of the spinal subarachnoid space. Physiol Behav. 1976;17(6):1031-6.

17. Linden DR, Sharkey KA, Mawe GM. Enhanced excitability of myenteric AH neurones in the inflamed guinea-pig distal colon 2003. 589-601.

18. Mallory B, Steers WD, Groat WCD. Electrophysiological study of micturition reflex in rats. Am J Physiol. 1989;257(2 Pt 2):R410.

19. Parsons BA, Drake MJ. Animal models in overactive bladder research. Handb Exp Pharmacol. 2011;202(202):15.

20. Vera PL, Nadelhaft I. Conduction velocity distribution of afferent fibers innervating the rat urinary bladder. Brain Res. 1990;520(1-2):83.

21. Su TH, Wang KG, Hsu CY, Wei HJ, Hong BK. Prospective comparison of laparoscopic and traditional colposuspensions in the treatment of genuine stress incontinence. Acta Obstet Gynecol Scand. 1997;76(6):576.

22. Vera PL, Nadelhaft I. Afferent and sympathetic innervation of the dome and the base of the urinary bladder of the female rat. Brain Res Bull. 1992;29(5): 651-8.

23. Bader C, Macleish P, Schwartz E. A voltage-clamp study of the light response in solitary rods of the tiger salamander. J Physiol Lond. 1979;296: $1-26$.
24. Difrancesco D, Tortora P. Direct activation of cardiac pacemaker channels by intracellular cyclic AMP. Nature. 1991;351(6322):145.

25. Doan TN, Kunze DL. Contribution of the hyperpolarization-activated current to the resting membrane potential of rat nodose sensory neurons. J Physiol. 1999;514(( Pt 1)(1)):125.

26. Mayer M, Westbrook G. A voltage-clamp analysis of inward (anomalous) rectification in mouse spinal sensory ganglion neurones. J Physiol Lond. 1983;340:19-45.

27. Pape HC. Queer current and pacemaker: the hyperpolarization-activated cation current in neurons. Annu Rev Physiol. 1996;58(1):299.

28. Funahashi M, Mitoh Y, Kohjitani A, Matsuo R. Role of the hyperpolarizationactivated Cation current ( $\mathrm{l} \mathrm{h}$ ) in pacemaker activity in area Postrema neurons of rat brain slices. J Physiol. 2003;552(1):135-48.

29. Rusznák Z, Pál B, Kőszeghy Á, Fu Y, Szücs G, Paxinos G. The hyperpolarization-activated non-specific cation current (I h ) adjusts the membrane properties, excitability, and activity pattern of the giant cells in the rat dorsal cochlear nucleus. Eur J Neurosci. 2013;37(6):876-90.

\section{Publisher's Note}

Springer Nature remains neutral with regard to jurisdictional claims in published maps and institutional affiliations.

Ready to submit your research? Choose BMC and benefit from:

- fast, convenient online submission

- thorough peer review by experienced researchers in your field

- rapid publication on acceptance

- support for research data, including large and complex data types

- gold Open Access which fosters wider collaboration and increased citations

- maximum visibility for your research: over $100 \mathrm{M}$ website views per year

At $\mathrm{BMC}$, research is always in progress.

Learn more biomedcentral.com/submissions 